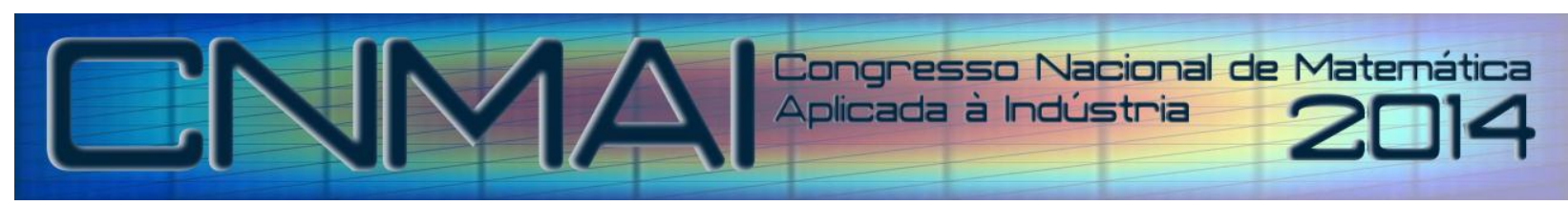

18 a 21 de novembro de 2014, Caldas Novas - Goiás

\title{
METODOLOGIA PARA OTIMIZAÇÃO DO PROJETO DE TRANSMISSÕES POR ENGRENAGENS CILINDRICAS EVOLVENTAL E APLICAÇÃO DE TÉCNICAS DE CORREÇÃO DE PERFIL
}

\author{
Paulo Sérgio Dias da Silva, paulodias83@live.com ${ }^{1}$ \\ Antônio Eustáquio de Melo Pertence, pertence@demec.ufmg.br ${ }^{1}$ \\ ${ }^{1}$ Universidade Federal de Minas Gerais, Departamento de Engenharia Mecânica, Av. Presidente Antonio Carlos 6.627, \\ Pampulha, Belo Horizonte, Minas Gerais, Brasil, 31270-901.
}

\begin{abstract}
Resumo: As engrenagens são elementos mecânicos fundamentais para os processos industriais. Estes componentes, quando bem dimensionados, cumprem suas funções de transmissão de potência e podem ter seu desempenho otimizado. Esta otimização é feita, basicamente, pelo ajuste dos perfis das engrenagens utilizando técnicas de deslocamento ou correção de perfil, propiciando nas mais variadas condições o aumento da capacidade de transmissão e a melhoria das condições de recobrimento e interferência. Por conseguinte o desenvolvimento de metodologias para otimização de projetos de engrenagens utilizando novas ferramentas torna-se essencial. $O$ objetivo deste trabalho é o desenvolvimento de uma metodologia para otimização do projeto de transmissões por engrenagens cilíndricas com aplicação da correção de perfil utilizando a geração virtual customizada de transmissões de engrenagens em condições de engrenamento previamente definidas através do software Catia $\mathbb{R}$. É feita uma comparação qualitativa considerando a alteração de parâmetros de engrenamento tais como ângulo de pressão, numero mínimo de dentes, tipos de correção de perfil (0 V0 e V), correção de perfil, grau de recobrimento, interferência e relação de redução, visando o processo de otimização. Utiliza-se a simulação numérica pelo método de elementos finitos para validar e auxiliar os cálculos analíticos tradicionais de tensões no pé do dente.
\end{abstract}

Palavras-chave: Projeto de engrenagens, Deslocamento de perfil, Geração virtual customizada de transmissões de engrenagens.

\section{INTRODUÇÃO}

Desenvolver equipamentos que facilite tarefas em seu dia a dia tem sido constante para o homem desde o início da humanidade. Muitas vezes estes possibilitam a multiplicação da força humana e as engrenagens desempenham papeis importantes e constituem num dos tipos de transmissões mais utilizados. Tal fato pode ser explicado pela grande variedade de tipos, faixas de aplicação e capacidades que podem ser utilizadas. As transmissões por engrenagens apresentam relações definidas de movimento utilizando rodas dentadas. Os dentes das engrenagens apresentam perfis específicos que procuram promover um engrenamento suave e cuja relação de transmissão permaneça constante. Os perfis evolventes de círculo estão entre os mais aplicados atualmente. A aplicação da técnica de correção de perfil que corresponde à utilização de diferentes setores da curva evolvente, possibilitando a melhoria de capacidade de carga $\mathrm{e}$ reduzindo o escorregamento entre os dentes das engrenagens. No projeto de transmissões por engrenagens normalmente se destacam dois critérios que se completam, a saber: o critério de resistência e o critério de vida. Dependendo da aplicação, um destes critérios poderá ser preponderante. Assim, no sentido de poder desenvolver um projeto de uma determinada transmissão por engrenagens cilíndricas considerando-se todas as condições de engrenamento existentes, é necessário o desenvolvimento de uma metodologia que venha validar e auxiliar os cálculos analíticos tradicionais, visando assim um processo de otimização. O objetivo deste trabalho é o desenvolvimento de uma metodologia para otimização do projeto de transmissões por engrenagens cilíndricas com aplicação da correção de perfil utilizando a geração virtual customizada de transmissões de engrenagens em condições de engrenamento previamente definidas através do software Catia®. Valério et al. (2010), mostra texto onde há aplicação de software para geração de desenhos e elementos de máquinas. É feita uma comparação qualitativa considerando a alteração de parâmetros de engrenamento tais como ângulo de pressão, numero mínimo de dentes, correção de perfil, grau de recobrimento, interferência e relação de redução, visando processo de otimização. Este processo pode ser realizado devido ao fato de que as engrenagens são 
elementos mecânicos cujas dimensões gerais e do perfil do dente, são definidos por relações matemáticas e de parametrização. É possível a comparação qualitativa do nível de tensões na base do dente considerando o adelgaçamento gerado pela escolha abaixo ou acima do número mínimo de dentes e sua variação com a alteração do ângulo de pressão, utilizando a simulação numérica pelo método de elementos finitos para validar e auxiliar os cálculos analíticos tradicionais.

\section{TRANSMISSÕES POR ENGRENAGENS}

As transmissões por engrenagens podem ser definidas como a ligação entre dois elementos que apresentam certa relação de movimento e podem ser classificadas quanto ao tipo de dente, posição relativa entre eixos, relação de transmissão, rendimento e tipos de engrenagens, entre estes se destacam: engrenagens cilíndricas, cônicas, parafuso sem fim coroa, de corrente, etc. As engrenagens cilíndricas podem ser retas ou helicoidais. O módulo é uma grandeza que relaciona as várias grandezas, tais como o diâmetro primitivo, número de dentes e ângulo da hélice. Os perfis dos dentes das engrenagens mais utilizados são os cicloidais e evolvente de círculo. Este último perfil pode ser expresso matematicamente e por isto é utilizado no desenvolvimento da técnica de correção de perfil. A Figura 1 ilustra o exposto acima.
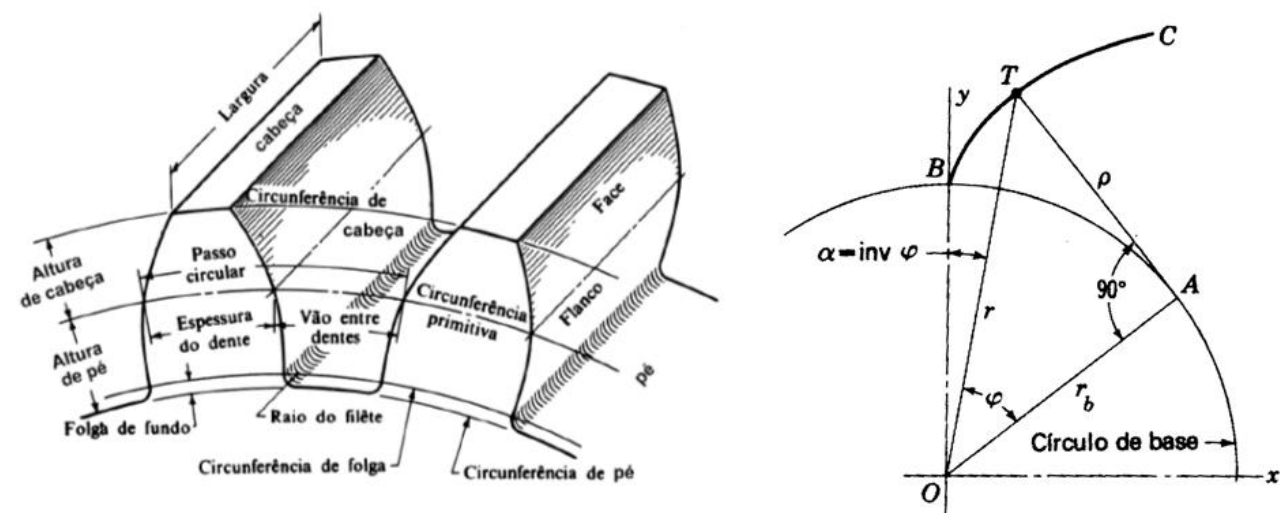

Figura 1. Nomenclatura dos dentes de engrenagens cilíndricas retas e curva esquemática do perfil evolvente de círculo.

A interferência é o fenômeno que acontece quando a cabeça do dente de uma engrenagem interfere provocando adelgaçamento no fundo do dente da outra engrenagem conjugada. Uma das causas da interferência é o uso de engrenagens com número mínimo de dentes abaixo do recomendado. O grau de recobrimento informa quantos pares de dentes se encontram acoplados num engrenamento ao mesmo tempo. As engrenagens cilíndricas helicoidais têm recobrimento maior que as engrenagens cilíndricas retas. A Figura 2 mostra o conceito de recobrimento e o fenômeno da interferência indicando que parte do perfil do dente da engrenagem é constituída por uma curva denominada trocoide. A Tabela 1 indica o número mínimo de dentes sem interferência em função do ângulo de pressão.

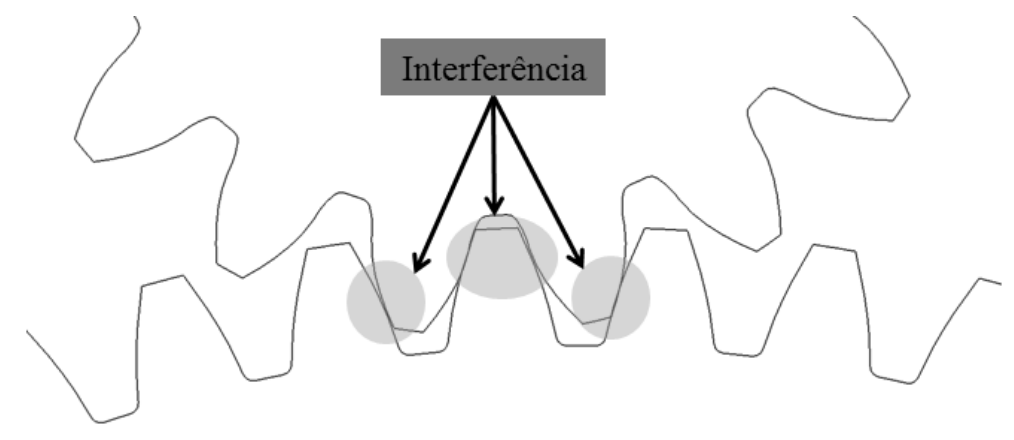

Figura 2. Fenômeno da interferência e conceito do recobrimento.

Para que se possam resolver os problemas de grandes relações de redução onde há possibilidade de interferência ou há necessidade de aumentar-se a capacidade de carga, mantendo-se a mesma distância entre centros utiliza-se a técnica de deslocamento ou correção de perfil. Esta técnica consiste basicamente em utilizar no perfil do dente, a parte da curva evolvente de círculo mais apropriada de acordo com o caso, conseguindo assim um melhor desempenho do engrenamento. A técnica de correção de perfil se apresenta através de equações capazes de relacionar todas as grandezas envolvidas e utiliza como parâmetro de quantificação o fator de perfil (x). A Figura 3 ilustra o conceito da técnica de correção de perfil. Souza et al. (2003), mostra a influência do ângulo de pressão em projetos de engrenagens. 
Normalmente consideram-se as forças atuantes no diâmetro primitivo da engrenagem e que todo o esforço de transmissão acontece em um só par de dentes, na linha de atuação do ângulo de pressão, embora o recobrimento mínimo aceito para as engrenagens cilíndricas retas seja de 1.2. Silveira et al. (2011), mostra estudos realizados para obtenção da tensão no pé do dente. O fator de concentração de tensão pode ser dado em função do número de dentes e do fator de correção do perfil.

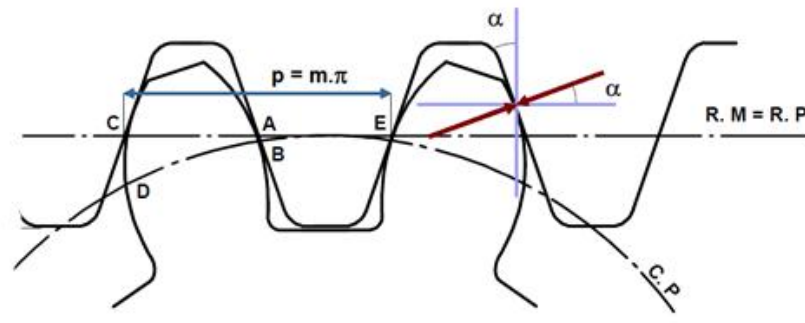

a) Geração do perfil evolvente.

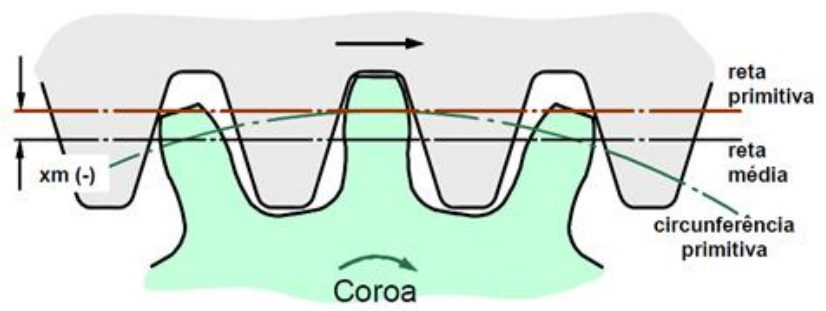

c) Correção negativa de perfil.

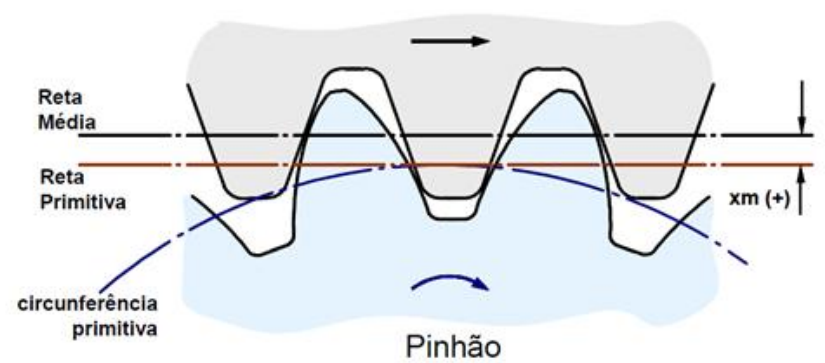

b) Correção positiva de perfil.

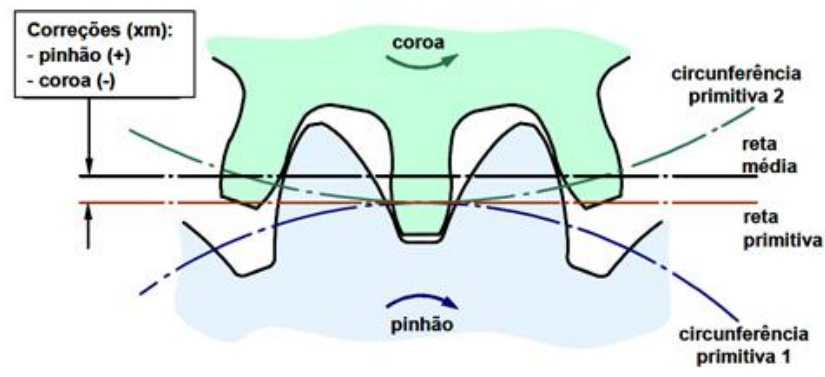

d) Correções positiva e negativa atuando conjuntamente.

Figura 3. Conceito da técnica da correção de perfil.

\section{METODOLOGIA}

Foram desenvolvidas inicialmente planilhas de cálculo em Excel® com equações relativas às relações geométricas das engrenagens cilíndricas retas visando auxilio e comparação com o modelo virtual gerado pelo software Catia ${ }^{\circledR}$. Vários trabalhos (Souza, 2003; Bezerra e Junior, 2001; Milovici e Turrin, 1983; Queiroz e Brazzalle, 1978) descrevem formas para gerar perfis de engrenagens, através do qual se baseia nos métodos analíticos. Este trabalho baseia-se nestes métodos. Foram considerados como dados de entrada o módulo, o número de dentes, o ângulo de pressão, o fator de correção de perfil, o ângulo da hélice e largura da engrenagem. A partir destes dados foram obtidas todas as grandezas gerais das engrenagens tais como diâmetro primitivo, diâmetro externo e diâmetro interno, diâmetro de base, passo e ângulo de simetria, etc. Considerou-se um modulo normal unitário e largura de $10 \mathrm{~mm}$ para todos os casos avaliados de engrenagens. Foram considerados neste estudo os seguintes ângulos de pressão no diâmetro primitivo: $14^{\circ} 30^{\prime}, 17^{\circ} 30^{\prime}$, $20^{\circ}, 22^{\circ} 30^{\prime}$ e $25^{\circ}$ que representam valores definidos por normas e industrialmente aplicados. Para cada ângulo de pressão considerado foi possível obter o número mínimo de dentes sem interferência, conforme pode ser visto na Tab. 1. Em seguida foram gerados os dados necessários para a construção da curva evolvente de círculo. Dependendo do caso foram utilizadas partes de uma curva trocoide para obtenção do seguimento do fundo e da região de adelgaçamento na base do dente. A Figura 4 ilustra planilha de cálculo desenvolvida em Excel $^{\circledR}$.

Tabela 1. Número mínimo de dentes sem interferência.

\begin{tabular}{|c|c|}
\hline Ângulo de Pressão - $\alpha$ (graus) & Número mínimo de dentes $Z_{\operatorname{mim}}$ \\
\hline $14^{\circ} 30^{\prime}$ & 32 \\
\hline $17^{\circ} 30^{\prime}$ & 22 \\
\hline $20^{\circ}$ & 17 \\
\hline $22^{\circ} 30^{\prime}$ & 14 \\
\hline $25^{\circ}$ & 11 \\
\hline
\end{tabular}



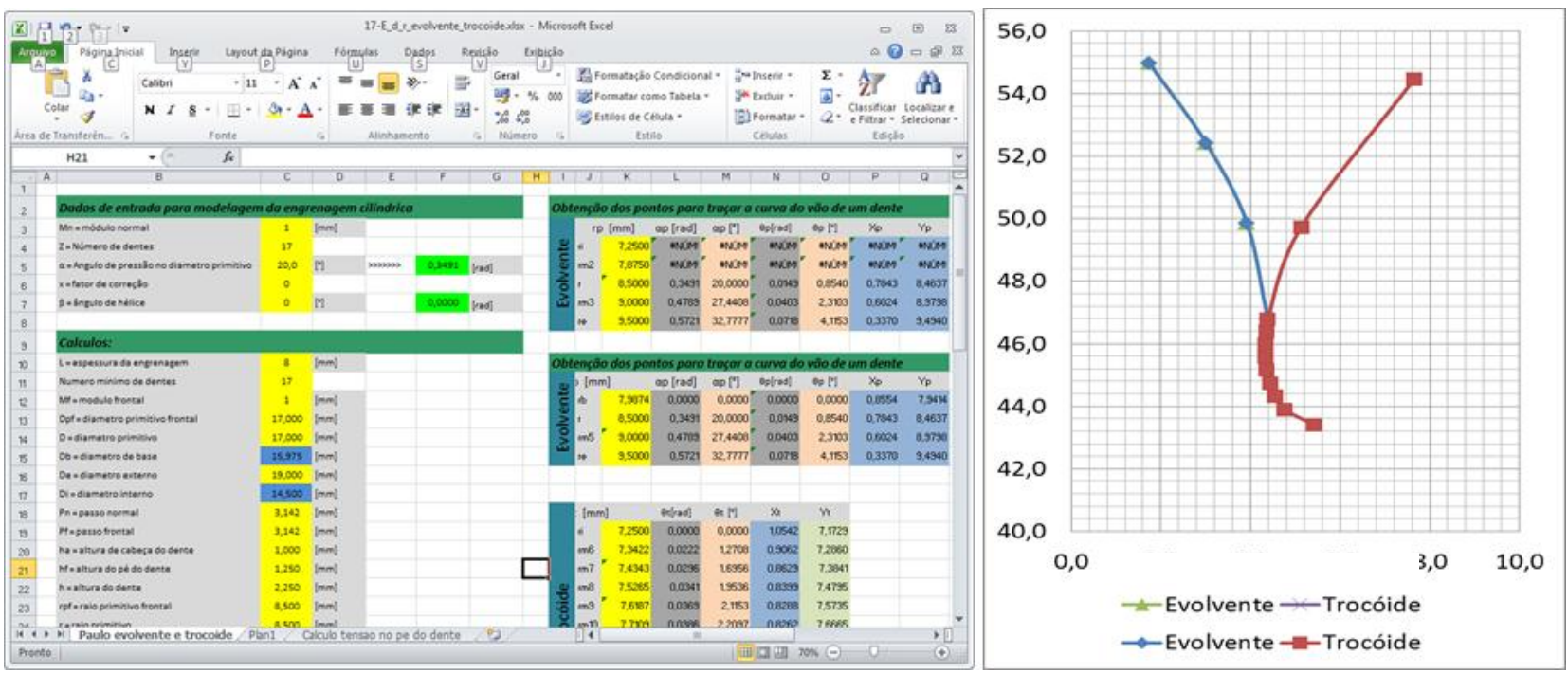

Figura 4. Planilha de cálculo com os dados de entrada e das grandezas gerais das engrenagens cilíndricas retas e pertil gerado do dente representando respectivamente as curvas evolvente e trocoide.

Para a modelagem de todos os casos estabelecidos, foram previamente desenvolvidos dois padrões de engrenagens cilíndricas retas utilizando o software Catia ${ }^{\circledR}$ V5 Release 19. Os modelos se direnciam pela existência ou não da curva trocoide junto perfil do dente da engrenagem. A existência da trocoide e assim do adelgaçamento do dente acontece quando o diâmetro interno de uma dada engrenagem cilíndrica reta for menor que o seu diâmetro de base. Assim se o sistema analisa os dados de entrada e faz a opção automática de um ou outro padrão.

A Figura 5 ilustra tela de geração virtual customizada de transmissões de engrenagens obtida através do software Catia ${ }^{\circledR}$ em conjunto com uma janela de interação com usuário para inserção dos dados básicos da engrenagem. Com este procedimento foi possível obter-se uma árvore com as instruções, onde são registradas as sequências da modelagem e uma tabela base onde ficam guardados os dados geométricos da engrenagem e que podem ser editados. Janelas de interação com o usuário foram criadas para facilitar a edição dos cálculos da geometria da engrenagem, através da utilização da linguagem Vbscript como indica a Fig. 6.

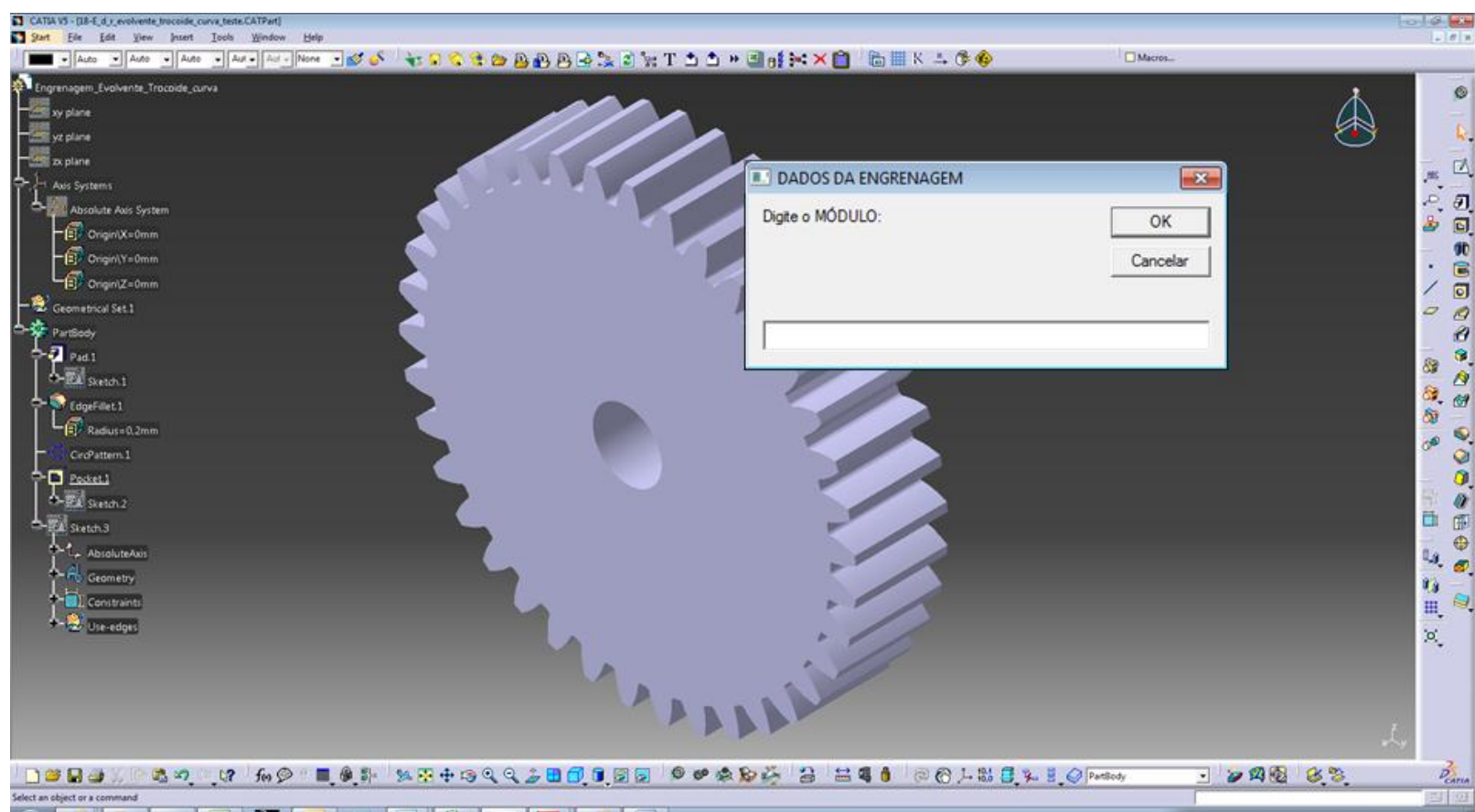

Figura 5. Modelo 3D obtido pela geração virtual customizada de transmissões de engrenagens através do software Catia ${ }^{\circledR}$. 


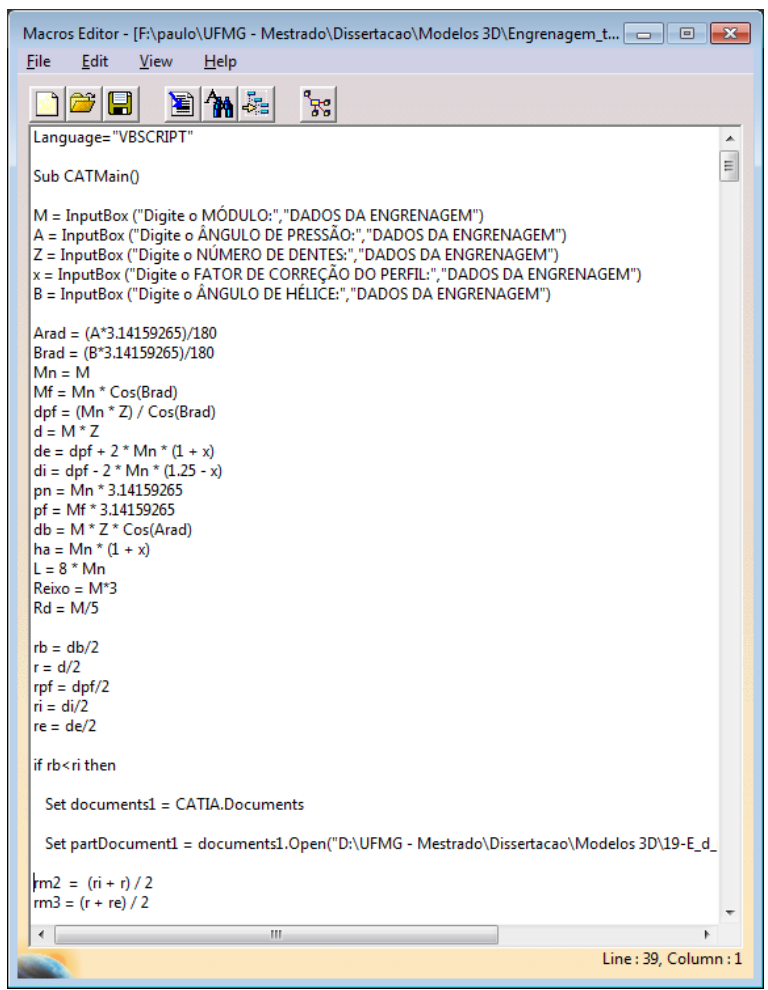

Figura 6. Janela de edição do arquivo em linguagem VBScript.

\section{RESULTADO E DISCURSÃO}

\subsection{Comparação do perfil do dente para vários módulos.}

Sabe-se que o módulo é uma grandeza que relaciona as várias grandezas das engrenagens tais como o diâmetro primitivo, número de dentes e ângulo da hélice. A Figura 7 indica a comparação entre os perfis de engrenagens cilíndricas retas considerando-se o mesmo número de dentes e um mesmo ângulo de pressão, variando-se apenas o módulo. Pode ser observado que a alteração da grandeza módulo influencia diretamente nas dimensões do dente e da engrenagem como um todo. Quanto maior o módulo considerado, maiores serão as dimensões da engrenagem e menores serão as tensões atuantes, sem influenciar na geometria do perfil.

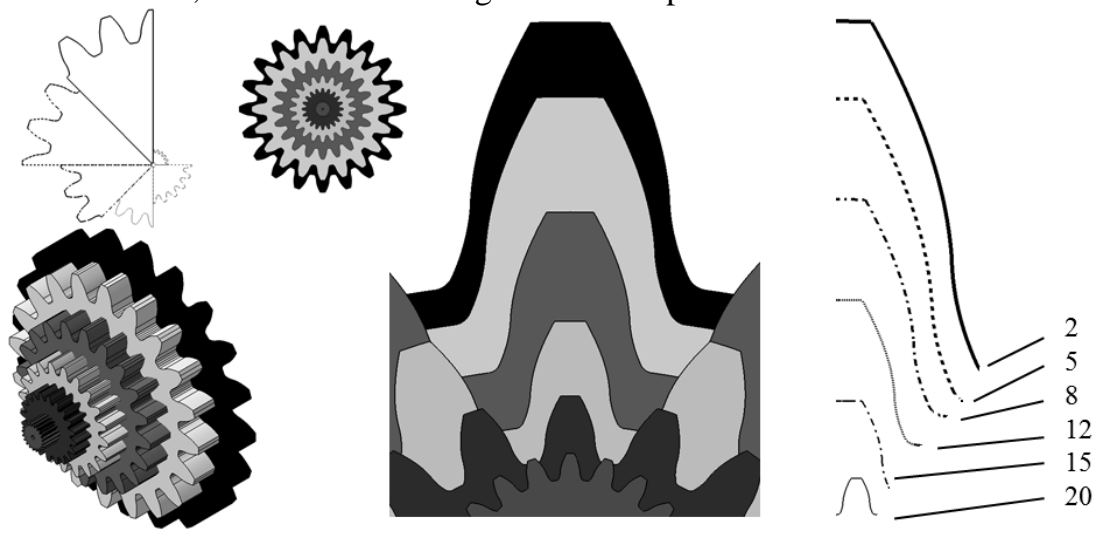

Figura 7. Comparação do perfil do dente para vários módulos (engrenagens cilíndricas retas com 20 dentes, ângulo de pressão de $20^{\circ}$ e módulos $2,3,5,8,10,12,15$ e 20 mm).

\subsection{Avaliação da influência da variação do ângulo de pressão no perfil do dente.}

Considerando-se engrenagens cilíndricas com o mesmo módulo e com ângulos de pressão de $14^{\circ} 30^{\prime}, 17^{\circ} 30^{\prime}, 20^{\circ}$, $22^{\circ} 30^{\prime}$ e $25^{\circ}$, cujo número de dentes seja igual ao número mínimo do ângulo de pressão de $14^{\circ} 30^{\prime}$ (32 dentes), observase uma redução do adelgaçamento com o aumento do angulo de pressão.

Considerando-se engrenagens cilíndricas com o mesmo módulo e com ângulos de pressão de $14^{\circ} 30^{\prime}, 17^{\circ} 30^{\prime}, 20^{\circ}$, $22^{\circ} 30^{\prime}$ e $25^{\circ}$, cujo número de dentes seja igual ao número mínimo do ângulo de pressão de $25^{\circ}$ ( 11 dentes), observa-se um aumento acentuado do adelgaçamento com a diminuição do angulo de pressão. A Figura 8 ilustra o exposto acima. 

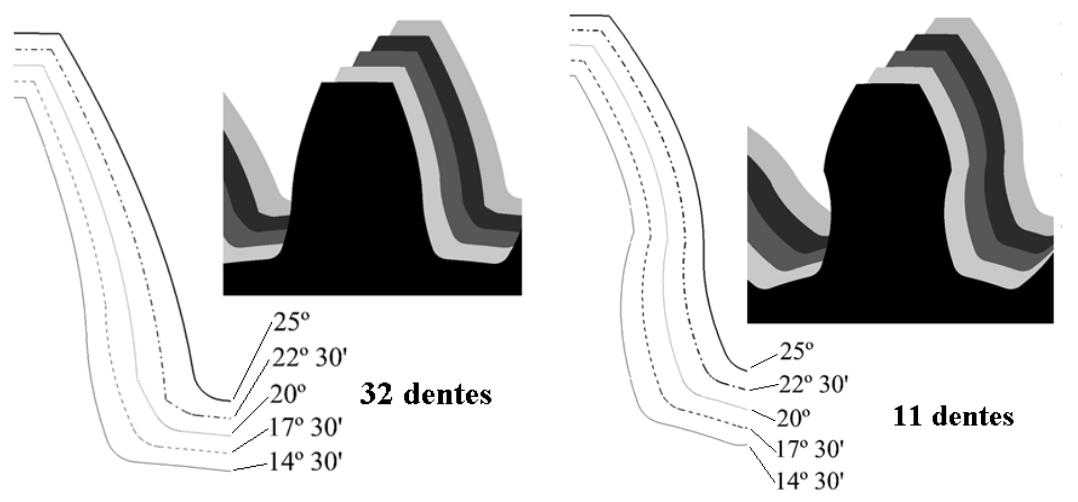

Figura 8. Comparação entre os perfis de engrenagens cilíndricas retas com 11 e 32 dentes para os ângulos de pressão de $14^{\circ} 30^{\prime}, 17^{\circ} 30^{\prime}, 20^{\circ}, 22^{\circ} 30^{\prime}$ e $25^{\circ}$ com o mesmo módulo.

Considerando-se o mesmo módulo, na medida em que o número de dentes de uma dada engrenagem cilíndrica é igual ou superior ao número mínimo de dentes, o efeito do adelgaçamento na base do dente será tanto menor, reduzindo o nível de concentração de tensões e, por conseguinte as tensões atuantes, quanto maior for o ângulo de pressão considerado. Na Figura 9 onde o número dentes da engrenagem é igual a 60, não se observam adelgaçamentos para qualquer ângulo de pressão, pois o número de dentes já é bem superior ao número mínimo de dentes sem interferência para qualquer ângulo de pressão considerado e os perfis destas engrenagens tendem ao perfil da cremalheira.

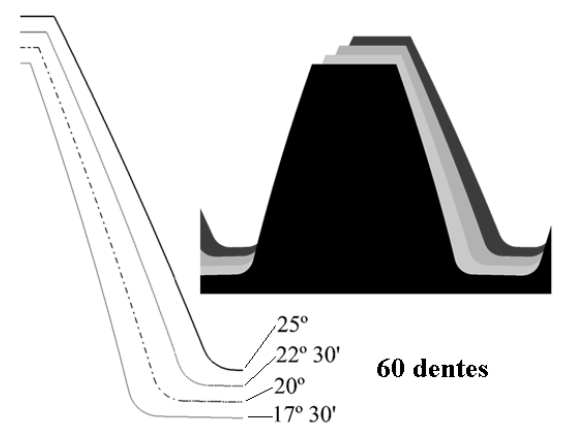

Figura 9. Comparação entre os perfis de engrenagens cilíndricas retas com 60 dentes para os ângulos de pressão de $17^{\circ} 30^{\prime}, 20^{\circ}, 22^{\circ} 30^{\prime}, 25^{\circ}$ para o mesmo módulo.

\subsection{Avaliação da influência do fator de correção no perfil do dente.}

A Figura 10 mostra os perfis do dente de uma engrenagem cilíndrica reta com 17 dentes, considerando os ângulos de pressão de $25^{\circ}$, sem fator de correção de perfil, com fatores de correção positivos $+0,25$ e $+0,5$ e negativos $-0,25$ e 0,5. Para as correções negativas há um adelgaçamento na base do dente com certo nível de concentração de tensões e o aumento das tensões atuantes e a diminuição das dimensões da engrenagem. Para as correções positivas há um alargamento da base e consequente diminuição das concentrações de tensões atuantes e um aumento das dimensões da engrenagem.
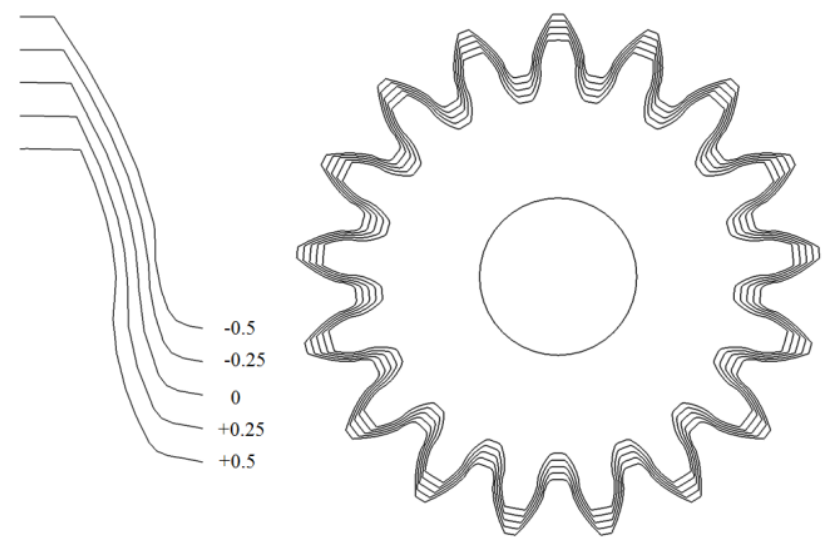

Figura 10. Comparação entre os perfis de engrenagens cilíndricas retas com 17 dentes, considerando os ângulos de pressão de $25^{\circ}$, sem fator de correção de perfil e vários fatores de correção de perfil. 
Quando se aplica um fator de correção de perfil positivo, haverá um aumento dos diâmetros de base, interno e externo, possibilitando o aumento da base e a redução do adelgaçamento. Assim uma engrenagem cilíndrica com o número de dentes igual ou abaixo do número mínimo de dentes para um dado ângulo de pressão, a presença do adelgaçamento poderá ser reduzido e, por conseguinte propiciar a redução do nível de tensões, aplicando a técnica de correção de perfil.

Por outro lado, quando se aplica um fator de correção de perfil negativo, haverá a diminuição dos diâmetros de base, interno e externo. Isto tende a reduzir a base e aumentar o adelgaçamento, porém se o numero de dentes é grande e o perfil já tende para um perfil de cremalheira e a influência da correção de perfil se torna pequena.

\subsection{Avaliação da influência do número de dentes na geometria da engrenagem e no perfil do dente.}

A Figura 11 indica a comparação entre as geometrias e perfil dos dentes de engrenagens cilíndricas retas com ângulo de pressão de $20^{\circ}$, com 12, 17, 22, e 45 dentes. Pode-se observar que o número de dentes influencia diretamente no tamanho da engrenagem que será tanto maior, quanto maior o seu número de dentes. Sabe-se que o módulo é uma grandeza que relaciona as várias grandezas das engrenagens tais como o diâmetro primitivo, número de dentes e ângulo da hélice. Observa-se que a grandeza módulo influencia exclusiva e diretamente na dimensão da engrenagem e do dente, alterando apenas em proporção, sem influência no perfil. Diferentemente do módulo e assim como a variação do ângulo de pressão, a variação do número de dentes pode aumentar ou diminuir o adelgaçamento no pé do dente e influenciar nas pressões exercidas no mesmo. Quanto maior o número de dentes, menor será o adelgaçamento. Pode-se observar que há um adelgaçamento maior na engrenagem de 12 dentes e diminui para a engrenagem de 17 dentes e consequentemente para a de 22 e 45 dentes.
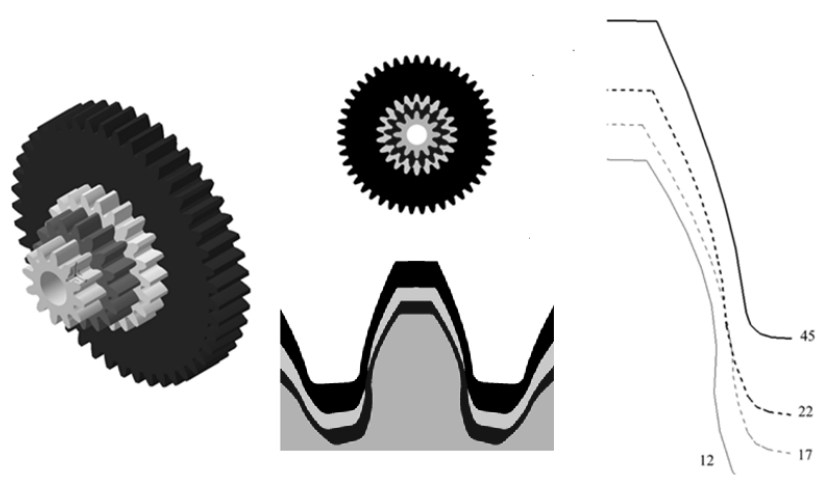

Figura 11. Comparação entre as geometrias e perfil de dentes de engrenagens cilíndricas retas com ângulo de pressão de $20^{\circ}$, com 12, 17, 22 e 45 dentes.

\subsection{Avaliação das tensões atuantes na base do perfil do dente.}

Para o cálculo das tensões atuantes na base do perfil do dente utilizou-se método analítico e simulação por elementos finitos, considerando-se situações com ou sem correção de perfil. As restrições e as ações foram posicionadas junto aos furos das engrenagens, sendo aplicado momento de torção de $10 \mathrm{Nm}$. Foi escolhido para todos os casos o material aço SAE 1020 a partir do banco de dados do software Catia ${ }^{\circledR}$. A Figura 12 ilustra a obtenção das tensões atuantes na base do perfil do dente a partir da geração virtual customizada de transmissões de engrenagens obtida no software Catia ${ }^{\circledR}$.

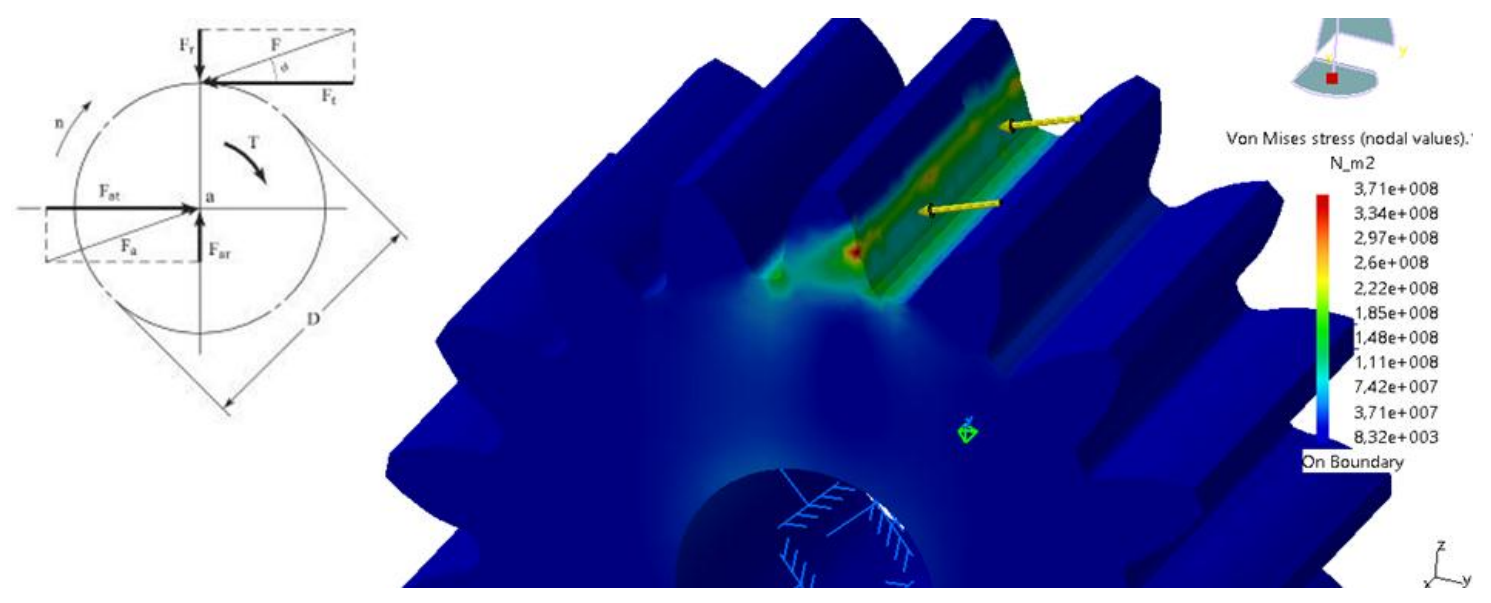

Figura 12. Tensões atuantes segundo Von Misses na base do perfil do dente. 
A Tabela 2 indica o resultado das tensões atuantes na base do perfil do dente, segundo Von Misses obtidas através da análise por elementos finitos em comparação aos valores obtidos ao método analítico, para engrenagens cilíndricas de 17 dentes com e sem aplicação da técnica de correção de perfil.

Tabela 2. Tensões atuantes na base do perfil do dente considerando o método analítico e por elementos finitos.

\begin{tabular}{|c|c|c|c|c|c|}
\hline $\begin{array}{c}\text { Número de } \\
\text { Dentes }\end{array}$ & $\begin{array}{c}\text { Ângulo de } \\
\text { pressão }\left(^{\circ}\right)\end{array}$ & $\begin{array}{c}\text { Fator de } \\
\text { Correção }\end{array}$ & $\begin{array}{c}\text { Fator de concentração } \\
\text { de tensão }\end{array}$ & $\begin{array}{c}\text { Método Analítico } \\
\left(\mathrm{N} / \mathrm{m}^{2}\right)\end{array}$ & $\begin{array}{c}\text { Método Elementos } \\
\text { Finitos }\left(\mathrm{N} / \mathrm{m}^{2}\right)\end{array}$ \\
\hline 17 & 20 & 0 & 2,90 & $3,41 \mathrm{E}+08$ & $3,71 \mathrm{E}+08$ \\
\hline 17 & 20 & +0.25 & 2,50 & $2,94 \mathrm{E}+08$ & $2,99 \mathrm{E}+08$ \\
\hline 17 & 20 & -0.25 & 3,60 & $4,24 \mathrm{E}+08$ & $4,53 \mathrm{E}+08$ \\
\hline
\end{tabular}

Os valores das tensões atuantes na base do perfil do dente com fator de correção de perfil negativo foram maiores do que aqueles encontrados com a aplicação do fator de correção positiva. Isto vem de encontro ao fato de que o processo de correção negativa provoca um maior adelgaçamento no perfil do dente e maior nível de tensões atuantes.

Observa-se que os valores alcançados para as tensões atuantes na base do perfil do dente, para tanto na aplicação do método analítico, quanto do método por elementos finitos, foram da mesma ordem de grandeza. Isto indica uma validação das condições de contorno adotadas no método de elementos finitos.

\section{AGRADECIMENTOS}

Os autores agradecem ao Programa de Pós-Graduação em Engenharia Mecânica da UFMG, ao Conselho Nacional de Desenvolvimento Científico e Tecnológico (CNPq), Fundação de Amparo à Pesquisa do Estado de Minas Gerais (FAPEMIG) e do Ministério da Educação (CAPES).

\section{REFERÊNCIAS}

Bezerra, R. A; Junior, F. I. S., "Projeto de Engrenagens de Dentes Retos com Perfil Evolvental", (COBEM) Congresso Brasileiro de Engenharia Mecânica, 2001.

Castro, R. M., "Critério de projeto para engrenagens helicoidais aplicadas em transmissões mecânicas veiculares", Dissertação de Mestrado apresentado à Escola Politécnica da Universidade de São Paulo (USP), 2005.

InduSoft Web Studio, "VBScript Reference Manual", 2007, http://www.indusoft.com.

Milovic, M.; Turrin, M. "Traçado do Perfil de Engrenagens Cilíndricas de Evolvente”, Mundo Mecânico, 1983.

Norton, R. L. "Projeto de máquinas, Uma abordagem integrada, 2 edição", pp. 622-629.

Queiroz, A. A.; Brazzalle, R. R., "Determinação do Perfil de Engrenagens de Evolvente com minicalculadoras", Mundo Mecânico, 1978.

Silveira, A. R. G. "Estudo da pressão de contato no pé do dente de uma engrenagem", 2011.

Souza, G. R., "Influência do ângulo de pressão em projetos de engrenagens", Dissertação de Mestrado apresentada à Universidade Estadual de Campinas (UNICAMP), 2003.

Valério, R.C.; Brito, J.N.; Souza, G. R., "Geração Automática de Desenhos na Plataforma CAD Solid Works a Partir de Células Paramétricas Desenvolvidas em Ambiente Visual Basic”, (SIMMEC) Simpósio de Mecânica Computacional , 2010.

\section{RESPONSABILIDADE AUTORAL}

Os autores são os únicos responsáveis pelo conteúdo deste trabalho. 


\title{
METHODOLOGY FOR OPTIMIZATION DESIGN OF TRANSMISSION BY EVOLVENTAL SPUR GEARS WITH PROFILE AND TEETH PROFILE MODIFICATION
}

\begin{abstract}
Paulo Sérgio Dias da Silva, paulodias83@live.com ${ }^{1}$ Antônio Eustáquio de Melo Pertence, pertence@ demec.ufmg.br ${ }^{1}$

${ }^{1}$ Federal University of Minas Gerais, Department of Mechanical Engineering, Presidente Antonio Carlos Avenue 6627, Pampulha, Belo Horizonte, Minas Gerais, Brazil, 31270-901.

Abstract: The gears are fundamental mechanical elements for industrial process. These components, when properly scaled, fulfill their duties of power transmission and can have their optimum performance. This optimization is done basically by fitting the profiles of gears using techniques of displacement or profile correction, providing in most varied conditions, increased transmission capacity and improving the coverage and interference. Therefore the development of methodologies for optimizing gear designs using new tools becomes essential. The objective of this work is to develop a methodology optimizing the design of cylindrical gear transmissions with application of profile correction using the customized virtual generation of transmissions gears with gearing conditions previously set by Catia $\circledast$ software. A qualitative comparison considering the modification of gearing parameters such as pressure angle, minimum number of teeth, types correction profile $(0 \mathrm{VO}$ and $\mathrm{V})$, Teeth profile modification, degree of coverage, interference and reduction ratio is taken. We use numerical simulation by finite element method to validate and support the traditional analytical calculations stress on the tooth.
\end{abstract}

Keywords: Design gears, Teeth profile modification, Customized virtual generating gears transmissions. 